\title{
Roles of White Blood Cells and Subtypes as Inflammatory Markers in Skin Cancer
}

\author{
Halit Baykan $^{1 *}$, Yasemin Benderli Cihan ${ }^{2}$, Kemal Ozyurt ${ }^{3}$
}

\begin{abstract}
Objective: Skin tumors are the most commonly seen cancer type worldwide. Regarding pathogenesis, it is thought that disruption of kinetics through $T$ lymphocyte-mediated development of chronic inflammation may be involved. The present study was intended to identify role of inflammatory cells such as neutrophils, monocytes and lymphocytes in the determination of risk for skin cancer. Materials and Methods: We retrospectively reviewed charts of 569 cases diagnosed as having primary skin tumors. Data regarding age, gender and histopathological subtype were recorded. Blood parameters studied on the day before surgery including WBCs, neutrophils, and lymphocyte counts, neutrophil:lymphocyte and neutrophil:monocyte ratios were also recorded. Two-hundred and two healthy individuals presented for check-up in an outpatient clinic were selected as the control group. Parameters studied in cases with skin cancer were compared to those healthy individuals. Findings: Of the cases with skin cancer, 401 were basal cell carcinoma (BCC) while 144 were squamous cell carcinoma (SCC) and 13 were malignant melanoma (MM). WBC, neutrophil and monocyte counts and the neutrophil:lymphocyte ratio were found to be lower in the patient group than in the healthy control group $(\mathbf{p}<0.001)$ while no significant difference was found in other parameters reviewed $(p>0.05)$. No significant difference was found in WBC, neutrophil, neutrophil: monocyte ratio according to gender $(p>0.05)$. Monocyte count was found to be $0.68 \pm 0.61$ in men and $0.55 \pm 0.25$ in women, indicating strong statistical significance $(p<0.001)$. WBC, neutrophil and monocyte values were highest in control group while lowest in BCC. When BCC and SCC groups were compared to controls, significant differences found $(\mathbf{p}<\mathbf{0 . 0 0 1})$. There were no significant differences in lymphocyte counts among groups (p=0.976). Neutrophil:lymphocyte ratios were 3.24 in BCC, 3.59 in SCC, 3.44 in MM and 5.06 in control group $(\mathbf{p}<0.001)$. Conclusions: In our study, it was found that there were significant differences in complete blood count, neutrophil, monocyte and neutrophil:lymphocyte levels among groups. Neutrophil: lymphocyte ratio was found to be lowest in BCC among skin cancers.
\end{abstract}

Keywords: Skin cancer - WBCs - neutrophils - lymphocytes - neutrophil:lymphocyte ratio

Asian Pac J Cancer Prev, 16 (6), 2303-2306

\section{Introduction}

The skin, largest organ in human body, exposes to all carcinogens for prolonged periods. Thus, skin cancers are most commonly seen type of malignancy in humans (Jemal et al., 2011). Annually, 800,000 new cases in US and 2.75 million new cases worldwide are diagnosed with increasing incidence by time (Marcil and Stern, 2000, Benderli Cihan et al., 2013). Again, skin tumors comprise one-third of all tumors treated in US (Parker et al., 1996).

The most prevalent malignant tumor of skin is basal cell carcinoma; followed by squamous cell, malign melanoma and tumors of skin attachments. Basal cell carcinoma comprises highest proportion among these tumors with highly variable biological behaviors. BCC has best prognosis while malign melanoma has poorest prognosis (Miller and Weinstock, 1994).

Risk for skin cancer development is found to be associated to age, gender, environmental factors, phenotypic features and immunity. However, pathogenesis hasn't been fully elucidated, although chronic inflammation is implied in the pathogenesis (Cancer, 2002; Benderli Cihan et al., 2013).

The aim of present study was to compare complete blood count, neutrophil, monocyte, lymphocyte, neutrophil: lymphocyte and neutrophil: monocyte parameters and to evaluate potential differences.

\section{Materials and Methods}

We retrospectively reviewed charts of 569 cases diagnosed as primary skin tumors and designated as Group 1. Two-hundred and two healthy individuals presented to check-up outpatient clinic were employed as controls and designated as Group 2. From the patient charts, data regarding age, gender and histopathological subtype were

${ }^{1}$ Department of Plastic and Reconstructive Surgery, ${ }^{2}$ Department of Radiation Oncology, ${ }^{3}$ Department of Dermatology, Kayseri Education and Research Hospital, Kayseri, Turkey*For correspondence: halitbaykan@gmail.com 
recorded. Blood parameters studied on the day before surgery including WBC, neutrophil, and lymphocyte counts, neutrophil: lymphocyte and neutrophil: monocyte parameters were also recorded. Parameters studied in cases with skin cancer were compared to those healthy individuals. The patients with missing data were excluded. Results of the cases with skin cancers and healthy individuals were compared statistically.

Data were analyzed by using SPSS for Windows version 15.0. Quantitative parameters were compared by using $\mathrm{t}$ test. Correlation analyses between categorical variables were performed by using Chi-square test with 95\% confidence interval. One-way variance analysis (oneway ANOVA) was used for comparisons between groups regarding mean values, standard deviation, lowest and highest intervals; while Duncan's test was used to detect groups causing difference. $\mathrm{p}<0.05$ was considered to be statistically significant.

\section{Results}

In the present study, cases with skin cancer were designated as group 1, while healthy individuals were designated as group 2. Of the subjects, 488 were men while 283 were women. In the group 1, there were BCC in 401 cases, SCC in 144 cases and MM in 13 cases. Mean age was $69.30 \pm 13.92$ years in group 1 and $62.57 \pm 10.59$ years in group 2 .

Table 1 presents WBC, neutrophil, monocyte, lymphocyte, neutrophil: lymphocyte and neutrophil: monocyte values in all groups. Mean WBC Values were $8.14 \pm 3.02$ and $8.46 \pm 1.30$ while mean monocyte values were $0.57 \pm 0.29$ and $0.81 \pm 0.85$ in groups 1 and 2 , respectively. Mean neutrophil values were 5.27 \pm 2.73 and $6.40 \pm 3.74$ while mean lymphocyte values were $2.16 \pm 1.68$ and $2.20 \pm 3.04$ in groups 1 and 2 respectively. When mean values were assessed, neutrophil: lymphocyte and neutrophil: monocyte ratio was found to be lower in the group 1. Significant differences were observed in complete blood count, neutrophil, monocyte, neutrophil: lymphocyte and age between groups $(\mathrm{p}<0.001)$.

Table 2 presents parameters according to gender. WBC, neutrophil and neutrophil: monocyte values were found to be higher in men compared to women, but no significant difference was detected. Neutrophil: lymphocyte ratio was $4.03 \pm 4.70$ in men and $3.34 \pm 5.51$ in women, indicating no significant difference $(\mathrm{p}=0.066)$.
Monocyte count was found to be $0.68 \pm 0.61$ in men while $0.55 \pm 0.25$ in women, indicating significant difference $(\mathrm{p}<0.001)$. Mean age ( \pm standard deviation) was found to be $66.73 \pm 12.60$ years in men and $68.92 \pm 14.69$ in women, indicating no significant difference.

Table 3 presents parameters according to cancer types. Highest WBC, neutrophil and monocyte values were found in the control group while lowest values were found in BCC. When BCC and SCC groups were compared to controls, significant differences were found $(\mathrm{p}<0.001)$. There were no significant differences in lymphocyte counts among groups $(\mathrm{p}=0.976)$. Neutrophil: lymphocyte ratios were 3.24 in BCC, 3.59 in SCC, 3.44 in MM and 5.06 in control group, indicating significant difference $(\mathrm{p}<0.001)$. When groups were assessed among groups, mean \pm standard deviation was $62.51 \pm 10.56$ in control group, 68.56 \pm 13.50 in BCC, $71.93 \pm 14.30$ in SCC and $62.07 \pm 16.83$ in $\mathrm{MM}$, indicating significant difference $(\mathrm{p}<0.001)$.

Table 1. Comparison of Complete Blood Count, Neutrophil, Lymphocyte, Monocyte, Neutrophil: Lymphocyte, Neutrophil: Monocyte Values among Groups

\begin{tabular}{lccr}
\hline Parameter & Cancer group Control group & $\mathrm{p}$ value \\
\hline Age $($ year $)$ & $69.30 \pm 13.9$ & $62.57 \pm 10.5$ & $<0.001$ \\
WBC $\left(\mathrm{x} 10^{3} \mu 1^{-1}\right)$ & $8.14 \pm 3.02$ & $9.61 \pm 4.95$ & $<0.001$ \\
Neutrophil $\left({\left.\mathrm{x} 10^{3} \mu 1^{-1}\right)}^{-1}\right)$ & $5.27 \pm 2.73$ & $6.40 \pm 3.74$ & 0.014 \\
Lymphocyte $\left(\mathrm{x} 10^{3} \mu 1^{-1}\right)$ & $2.16 \pm 1.68$ & $2.20 \pm 3.04$ & 0.829 \\
Monocyte $\left(\mu 1^{-1}\right)$ & $0.57 \pm 0.29$ & $0.81 \pm 0.85$ & $<0.001$ \\
Neutrophil/Lymphocyte & $3.34 \pm 4.59$ & $5.01 \pm 5.92$ & $<0.001$ \\
Neutrophil/Monocyte & $10.6 \pm 8.15$ & $13.00 \pm 34.5$ & 0.129 \\
\hline
\end{tabular}

*Values are expressed as mean \pm std. deviation

Table 2. Comparison of Complete Blood Count, Neutrophil, Lymphocyte, Monocyte, Neutrophil: Lymphocyte, Neutrophil: Monocyte Values and Ages According to Gender

\begin{tabular}{lcrr}
\hline Parameter & Male & Female & \multicolumn{1}{l}{ P } \\
\hline Age (year) & $66.9 \pm 12.6$ & $68.91 \pm 14.7$ & 0.043 \\
WBC $\left(\mathrm{x} 10^{3} \mu 1^{-1}\right)$ & $8.67 \pm 3.84$ & $8.30 \pm 3.41$ & 0.185 \\
Neutrophil $\left(\mathrm{x} 103^{3} \mu 1^{-1}\right)$ & $5.69 \pm 3.08$ & $5.36 \pm 3.06$ & 0.156 \\
Lymphocyte $\left(\mathrm{x} 10^{3} \mu 1^{-1}\right)$ & $2.09 \pm 2.08$ & $2.30 \pm 2.20$ & 0.189 \\
Monocyte $\left(\mu 1^{-1}\right)$ & $0.68 \pm 0.61$ & $0.55 \pm 0.25$ & $<0.001$ \\
Neutrophil/Lymphocyte & $4.02 \pm 4.67$ & $3.34 \pm 5.52$ & 0.069 \\
Neutrophil/Monocyte & $11.41 \pm 23.0$ & $11.0 \pm 9.01$ & 0.795 \\
\hline *Values are expressed as mean \pm std. deviation & &
\end{tabular}

Table 3. Comparison of Complete Blood Count, Neutrophil, Lymphocyte, Monocyte, Neutrophil: Lymphocyte, Neutrophil: Monocyte Values and Ages According to Cancer Types

\begin{tabular}{|c|c|c|c|c|c|}
\hline Parameter & $\mathrm{BCC}$ & $\mathrm{SCC}$ & $\mathrm{MM}$ & Control & $\mathrm{P}$ value \\
\hline Age (year) & $68.56 \pm 13.5^{24}$ & $71.93 \pm 14.3^{134}$ & $62.07 \pm 16.8^{2}$ & $62.51 \pm 10.6^{12}$ & $<0.001$ \\
\hline $\mathrm{WBC}\left(\mathrm{x} 10^{3} \mu \mathrm{l}^{-1}\right)$ & $7.97 \pm 2.67^{4}$ & $8.48 \pm 3.73^{4}$ & $9.41 \pm 3.83$ & $9.66 \pm 4.9712$ & $<0.001$ \\
\hline Neutrophil(x103 $\left.\mu 1^{-1}\right)$ & $5.10 \pm 2.39^{4}$ & $5.58 \pm 3.43^{4}$ & $6.31 \pm 3.43$ & $6.45 \pm 3.75^{12}$ & $<0.001$ \\
\hline Lymphocyte $\left(\mathrm{x} 10^{3} \mu \mathrm{1}^{-1}\right)$ & $2.18 \pm 1.85$ & $2.10 \pm 1.13$ & $2.18 \pm 0.94$ & $2.20 \pm 3.06$ & 0.976 \\
\hline $\operatorname{Monocyte}\left(\mu 1^{-1}\right)$ & $0.56 \pm 0.29^{4}$ & $0.59 \pm 0.27^{4}$ & $0.67 \pm 0.40$ & $0.81 \pm 0.86^{12}$ & $<0.001$ \\
\hline Neutrophil/Lymphocyte & $3.24 \pm 4.91^{4}$ & $3.59 \pm 3.70^{4}$ & $3.44 \pm 2.62$ & $5.06 \pm 5.95^{12}$ & $<0.001$ \\
\hline Neutrophil/Monocyte & $10.41 \pm 7.96$ & $10.95 \pm 8.43$ & $12.97 \pm 10.5$ & $13.11 \pm 34.8$ & 0.418 \\
\hline
\end{tabular}

*Values are expressed as mean \pm std. deviation; ${ }^{1234}$ The groups in the same column with different number are statistically significant (p<0.05) 


\section{Discussion}

As known, skin is the largest organ in human body. Thus, it exposes to carcinogens more than other organs. Today, incidence of skin cancers is progressively increasing in the community. BCC has highest incidence; followed by SCC and MM (Jemal et al., 2011). As similar, $\mathrm{BCC}$ was seen most commonly in our clinic; followed by SCC and, to a lesser extent, MM.

Tumor should have to rearrange micro-environment in order to proliferate at localization site. It can use immune and non-immune cells while doing this. These cells include lymphocytes, natural killer (NK) cells, macrophages, endothelial cells and pericytes (Coussen and Werb, 2002).

Neutrophils and poylmorphonuclear leukocytes (PNL) play important roles in elimination of microorganisms and wound healing (Nathan, 2006). If inflammation is present in the body due to any reason (infection, trauma or tumoral differentiation), neutrophil and PNL presence or increase at inflammation site are common. Several proteins are released to inflammation site from activated neutrophils. These proteins have major effects on formation of immune response (Nathan, 2006).

It is apparent that cancer has some effect on peripheral blood cells. Causes underlying increased neutrophil and monocyte haven't been fully understood yet. However, tumoral cells secrete several cytokines and chemokines. Eventually, these cells lead neutrophil and monocyte proliferation. Toxic granules in the cytoplasm of neutrophils are effective as one of the immunological defense mechanisms in cancer cells. In addition, vascular endothelial growth factor and IL-8 secreted from neutrophils are triggers of cancer-related angiogenesis. Normally, monocytes exist in blood and dendritic cells when differentiated or as tissue macrophages due to inflammation or tumor development by effects of cytokines and chemokines. Tumor-associated macrophages (TAM) in monocytes are important components of inflammatory infiltrates of neoplastic tissues. In the presence of tumor, TAM has two opposite effects. First is killing of neoplastic cells via activating IL-2, interferon and IL-12. Second is production of several potent angiogenic growth factors, cytokines and proteases that enhance tumor progression (Coussens and Werb, 2002; Bo Wook et al., 2012; Sparmann and Bar-Sagi, 2004; Mantovani et al., 2008).

In the development of malignancy, it is known that chronic inflammation accounts from development epithelial cancers including stomach liver, colon, lung, pancreas, esophagus, bladder and biliary tract cancers (Ardies, 2003; Wislez, et al., 2003; Margolis, 2007). Although reason or underlying mechanism is unclear, it is suggested that toxic granules in the cytoplasm of neutrophils account from inflammation in neoplastic tissues through activation of monocytes (Coussens and Werb, 2002; Nathan, 2006; Fridlender and Albelda, 2012). In the literature, relationship between inflammation and neutrophil in cancer is being intensively investigated.

Among skin cancers, BCC has best prognosis while SCC and MM have worse prognosis. In addition, recognition and treatment seems to be more readily as these cancers display visible symptoms over skin. Besides, there are markers for early diagnosis in many cancers but not in skin cancers. In many previous studies, neutrophil, neutrophil: lymphocyte and neutrophil monocyte levels were investigated and prognostic implications have been made from neutrophil ratios (Parker, 1996). We planned this study to identify parameters that could be marker for early detection or provide information regarding prognosis.

In our study, neutrophil: lymphocyte and neutrophil: monocyte ratios were found to be low in cancer groups. When groups were assessed regarding complete blood count, neutrophil, monocyte, neutrophil: lymphocyte values and age, significant differences were detected. Clinical studies suggested that presence of neutrophilia is a sign of poor prognosis in cancer cases. In previous studies, it was shown that prognosis was poorer in bronchoalveolar and renal cell cancers with high PNL levels. IL-8 levels indicate PNL accumulation which shows decreased life expectancy (Gregory and Houghton, 2011). In our study, PNL level was found to be high in SCC and MM with poor prognosis and lowest in BCC that has better prognosis.

Again, neutrophil: leukocyte ratio was found to be high in MM and SCC and low in BCC. In agreement with literature, these results showed that neutrophil and neutrophil: leukocyte values were increased by increasing malignancy in skin cancers. However, on contrary to literature, neutrophil: leukocyte ratio and neutrophil count were found to be higher in control group compared to cancer group. This finding indicates that immune system is normal in control group. In cancer cases, especially in those with higher malignancy, immune system is disrupted. In addition to defensive roles, immune system cells secrete cytokines that prepare micro-environment needed for cancer development (Coussens, 2002; Bo Wook, 2012; Sparmann , Bar-Sagi, 2004; Mantovani, et al, 2008). In conclusion, spread and growth of cancer are facilitated, causing poorer prognosis. When assessing cancer cases, increasing neutrophil and neutrophil: leukocyte ratio can provide an important clue for worsening prognosis.

In conclusion, results obtained show us that neutrophil and neutrophil: leukocyte ratio can be an important marker for demonstration of malignancy and its prognosis and in follow-up in cases with skin cancer.

\section{References}

Ardies CM (2003). Inflammation as cause for scar cancers of the lung. Integr Cancer Ther, 2, 238-46.

Benderli Cihan Y, Baykan H, Kavuncuoglu E, et al (2013). Relationships between skin cancers and blood groups -link between non-melanomas and $\mathrm{ABO} / \mathrm{Rh}$ factors. Asian Pac J Cancer Prev, 14, 4199-203.

Bo Wook Kim, Young Eun Jeon, Hanbyoul Cho, et al (2012). Pre-treatment diagnosis of endometrial cancer through a combination of CA125 and multiplication of neutrophil and monocyte. J Obstet Gynaecol Res, 38, 48-56.

Cancer incidence in five continents (2002). Lyon: International agency for research on cancer, Vol VIII, p. 234-240

Coussens LM, Werb Z (2002). Inflammation and cancer. Nature, 420, 860-7.

Fridlender ZG, Albelda SM (2012). Tumor-associated neutrophils: friend or foe? Carcinogenesis, 33, 949-55. 
Halit Baykan et al

Gregory AD, Houghton AM (2011). Tumor-Associated Neutrophils: New Targets for Cancer Therapy. Cancer Res, 71, 2411-6.

Jemal A, Bray F, Center MM, et al (2011). Global cancer statistics. CA: Cancer J Clin, 61, 69-90.

Mantovani A, Allavena P, Sica A, Balkwill F (2008). Cancerrelated inflammation. Nature, 454, 436-444.

Marcil I, Stern RS (2000). Risk of developing a subsequent non-melanoma skin cancer in patients with a history of nonmelanoma skin cancer. Arc Dermal, 136, 1524-30.

Margolis KL, Rodabough RJ, Thomson CA, Lopez AM, McTiernan A (2007). Prospective study of leukocyte count as a predictor of incident breast, colorectal, endometrial, and lung cancer and mortality in postmenopausal women. Arch Intern Med, 167, 1837-44.

Mantovani A, Allavena P, Sica A, Balkwill F (2008). Cancerrelated inflammation. Nature, 454, 436-44.

Miller DL, Weinstock MA (1994). Cancer head and neck-2 nonmelanoma skin cancer in the United States; incidence. $J$ Am Acad Dermatol, 30, 774.

Nathan C (2006). Neutrophils and immunity: challenges and opportunities. Nat Rev Immunol, 6, 173-82.

Parker S, Tong T, Bolden S, et al (1996). Cancer statistics 1996. Cancer J Clin, 4, 61-5.

Sparmann A, Bar-Sagi D (2004). Ras-induced interleukin-8 expression plays a critical role in tumor growth and angiogenesis. Cancer Cell, 6, 447-58.

Wislez M, Rabbe N, Marchal J, et al (2003). Hepatocyte growth factor production by neutrophils infiltrating bronchioloalveolar subtype pulmonary adenocarcinoma: role in tumor progression and death. Cancer Res, 63, 1405-12. 